\title{
Surveillance of Echinococcus tapeworm in coyotes and domestic dogs in Winnipeg, Manitoba
}

\author{
CCK Tse ${ }^{1}$, J Bullard ${ }^{1,2,3}$, R Rusk ${ }^{1,4,5}$, D Douma ${ }^{6}$, PJ Plourde $1,2,5,7 *$
}

\section{Abstract}

Background: The Echinococcus species, including E. multilocularis and E. canadensis, are tapeworms that primarily infect canids such as dogs, foxes and coyotes, but which can also infect humans. In humans, E. multilocularis can cause alveolar echinococcosis; a serious condition that mimics metastatic malignancy and has a poor prognosis. It is known that coyotes in rural Manitoba are infected with Echinococcus species, but it is not known if coyotes in peri-urban areas are also infected.

Objectives: To document and map Echinococcus species in wild canids and domestic dogs in Winnipeg, Manitoba (Canada).

Methods: There were 169 fecal samples collected between April 18 and June 1, 2018. These included 44 samples of domestic dog feces, 122 of coyote scat, one of fox scat and two of coyote colonic tissue specimens. Samples were frozen $\left(-80^{\circ} \mathrm{C}\right)$ for at least 72 hours to inactivate tapeworm ova. Polymerase chain reaction analyses of E. multilocularis and E. canadensis were performed on all frozen samples.

Results: Echinococcus multilocularis-positive samples were detected in nine (10.6\%) of 85 locations, with one positive sample in a suburban Winnipeg dog park and two positive samples in a popular provincial park. No dog samples were positive for E. multilocularis; one sample was positive for E. canadensis. In contrast, nine coyote samples (7.3\%) were positive for E. multilocularis and eight samples (6.5\%) were positive for E. canadensis. The one fox sample was positive for each. Overall, six samples (3.6\%) were positive for both infections.

Conclusion: This is the first confirmation of the presence of E. multilocularis in coyote feces in the metropolitan area of Winnipeg, Manitoba. In light of the risk this could pose to domestic dogs and human health, periodic surveillance that maps the distribution of this tapeworm could inform the need for additional public health actions.

Suggested citation: Tse CCK, Bullard J, Rusk R, Douma D, Plourde PJ. Surveillance of Echinococcus tapeworm in coyotes and domestic dogs of Winnipeg, Manitoba. Can Commun Dis Rep 2019;45(7/8):171-6.

https://doi.org/10.4745/ccdr.v45i78a01

Keywords: alveolar echinococcosis, hydatid disease, zoonotic diseases, coyotes, foxes, Echinococcus tapeworms, domestic dogs
This work is licensed under a Creative Commons Attribution 4.0 International License.

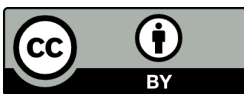

Affiliations

${ }^{1}$ Max Rady College of Medicine, Rady Faculty of Health Sciences, University of Manitoba, Winnipeg, $\mathrm{MB}$

${ }^{2}$ Department of Medical Microbiology and Infectious Diseases, Rady Faculty of Health Sciences, University of Manitoba, Winnipeg, MB

${ }^{3}$ Cadham Provincial Laboratory, Winnipeg, MB

${ }^{4}$ Public Health Branch, Manitoba Health, Seniors and Active Living, Winnipeg, MB

${ }^{5}$ Department of Community Health Sciences, Rady Faculty of Health Sciences, University of Manitoba, Winnipeg, MB

${ }^{6}$ Animal Health and Welfare Branch, Manitoba Agriculture, Winnipeg, MB

${ }^{7}$ Population and Public Health Program, Winnipeg Regional Health Authority, Winnipeg, MB

*Correspondence: pplourde@wrha.mb.ca 


\section{Introduction}

Echinococcus species are tapeworms that primarily infect canid species. The two predominant species that have been found in Canada are E. multilocularis and E. canadensis. The sylvatic lifecycle of Echinococcus tapeworms includes coyotes and other canids as definitive hosts and rodents as intermediate hosts. Humans, however, can be incidental (or dead-end) hosts (Figure 1). E. multilocularis can cause a serious disease in humans: alveolar echinococcosis $(1,2)$. This infection, which behaves like a metastatic malignancy, has a high case-fatality rate and requires radical surgical and long-term anthelminthic treatment (3). Although a more benign infection in humans, E. canadensis is also maintained via a sylvatic lifecycle thatincludes wolves, coyotes, and dogs as definitive hosts and caribou, moose and elk as intermediate hosts. In humans, as incidental hosts, the predominant clinical presentation is cystic hydatid disease primarily in the liver, which may require surgical intervention that carries the risk of severe anaphylaxis should cyst rupture occur (2). These two species are not infrequently found together given they share definitive canid hosts. Echinococcus species tapeworms are asymptomatic and cause no disease in canids, and they are hard to detect, as the worms are only 1-7 $\mathrm{mm}$ in length.

\section{Figure 1: Lifecycle of Echinococcus multilocularis}

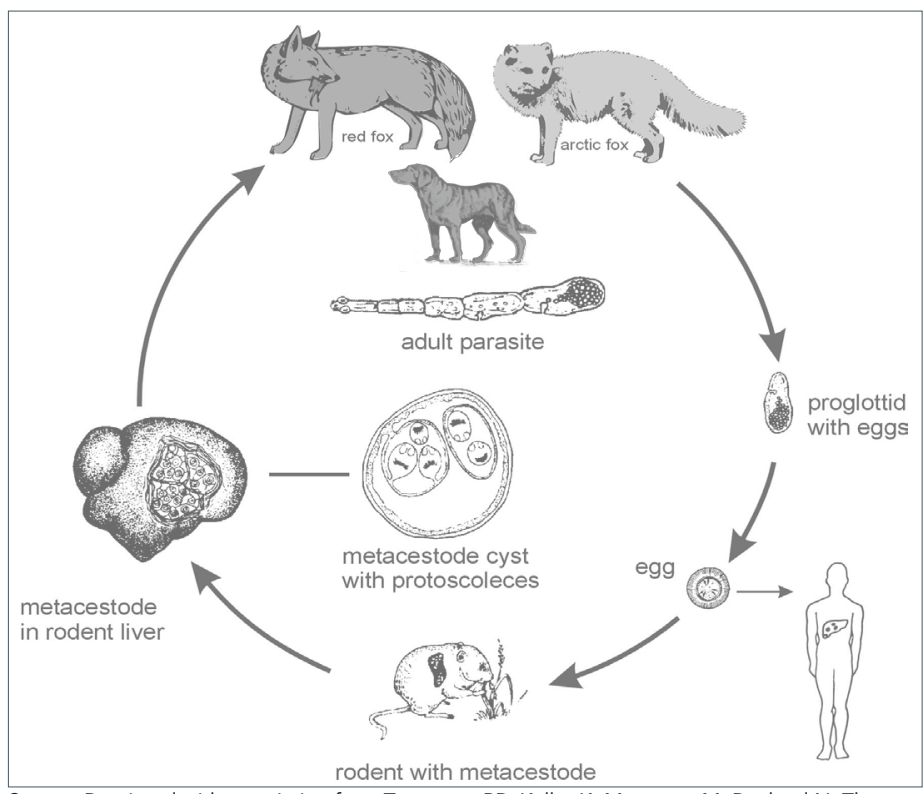

Source: Reprinted with permission from Torgerson PR, Keller K, Magnotta M, Ragland N. The Global Burden of Alveolar Echinococcosis. PLoS Negl Trop Dis 2010;4(6): e722

The World Health Organization and the Food and Agriculture Organization of the United Nations have ranked alveolar echinococcosis as the third most important food-borne parasitic disease of global importance (4). As there is no cost-effective way to eliminate E. multilocularis in the sylvatic lifecycle, surveillance is important for human risk assessment.

\section{How humans become infected}

Echinococcus multilocularis ova are quite resistant and can survive and remain infective for nearly one year under favorable conditions (5). As the ova are sticky, they can adhere to the fur of dogs, wild vegetation and garden produce that is grown in feces-contaminated soil $(2,6,7)$. Transmission to humans typically occurs when the hand comes in contact with water, soil, fur or objects contaminated with host feces, followed by inadvertent hand to mouth transfer of the ova. Following human ingestion of E. multilocularis ova, there is a long clinical latency period; from five to 15 years. Early symptoms include abdominal pain, which is followed by jaundice and eventually by severe hepatic dysfunction $(2,8)$. Alveolar echinococcosis is often mistaken for a neoplastic growth, given its propensity for widespread organ infiltration with metastases (2). E. multilocularis may also imitate other diseases, including hepatic carcinoma, cirrhosis and tuberculosis, which may lead to improper diagnostic testing and delayed treatment $(3,9,10)$. Diagnosis is confirmed based on a combination of clinical findings, epidemiological data, imaging, histopathology and/or nucleic acid detection and serology (8). Recommended treatment is radical surgical resection of the parasitic lesion, which in early stages may lead to a complete cure; however, surgical resection can sometimes be incomplete due to the diffuse or undetected spread of the parasite $(2,8)$. As such, post-surgical anthelminthic chemotherapy is recommended for at least two years followed by a minimum of 10 years of close monitoring (8). If untreated, mortality is $90 \%$ within 10 years of onset of symptoms (11). Early diagnosis and proper treatment has been shown to improve survival (12).

\section{Echinococcus multilocularis in Canada}

Echinococcus tapeworms have been documented in canids in Canada for many years. A study on the prevalence of E. multilocularis in Manitoba canid species, performed 40 years ago in Riding Mountain National Park, found that almost one quarter of all coyotes sampled were infected (13). More recently in 2009, an European strain of E. multilocularis was discovered in central British Colombia in a domestic dog with no history of travel outside Canada, with further research suggesting the possible establishment of this new strain in the local wildlife $(14,15)$. There may be evidence of a similar strain in the wildlife of Saskatchewan (16). Surveys in Calgary and Edmonton published in 2012 determined that E. multilocularis was present in the wild canid populations of those cities (1). Furthermore, in Ontario, recent detections of alveolar echinococcosis in several domestic dogs with no known travel history outside of Ontario suggest that $E$. multilocularis tapeworms may have established a foci in southwestern Ontario as well $(17,18)$.

Echinococcus tapeworms and cases of human alveolar echinococcosis were first reported in the 1930s in Manitoba and Alberta (19). More recently, in November 2017, a pediatric 
patient from Manitoba was identified with disseminated alveolar echinococcosis infection (personal communication November 23, 2017, Dr. Sergio Fanella). Similarly, in 2018, several human cases of alveolar echinococcosis were detected in Alberta (personal communication June 5, 2018, Dr. Stan Houston).

With increasing urbanization and sightings of coyotes in urban and suburban areas, E. multilocularis may be brought into close proximity to domestic dogs and humans $(20,21)$. In light of these recent human cases, and the sightings of coyotes in and around Winnipeg (20), a survey of wild canids to look for the presence of Echinococcus species was conducted.

The aim of this study was to determine the extent of Echinococcus species ova, especially E. multilocularis, in coyote, fox and domestic dog feces found in the metropolitan area around Winnipeg, and to perform geospatial mapping to determine at-risk areas.

\section{Methods}

\section{Collection sites}

The Winnipeg metropolitan area (WMA) was defined as Census Metropolitan Area 602 based on the 2016 census profile from Statistics Canada (22). This consists of the urban area, demarcated by the circumferential perimeter highway surrounding Winnipeg and the peri-urban area that lies outside the perimeter highway and within the WMA. Eighty-five collection sites were identified that covered a wide area of the WMA, including areas frequented by humans and domestic dogs as well as areas with known coyote sightings.

\section{Sample collection}

Fecal samples were collected between April 18 and June 1, 2018. Samples from domestic dogs were collected differently from the other canid samples. Dog samples were collected from dog parks or submitted directly by dog owners. Two drop-off locations were offered to volunteers who submitted fecal samples from domestic dogs.

Samples from wild canids (mostly coyotes) were predominantly collected by driving along remote roads near coyote habitat based on local knowledge of coyote sightings. Coyote samples were identified via characteristics of the scat sample including shape, texture, presence of hair and bone belonging to small animals as well as supporting features including coyote tracks, nearby coyote dens and previous sightings (20). Similarly the fox scat sample was differentiated by the local conservation officer by its smaller size and the fact there had been known fox sightings nearby.

All fecal samples were picked up individually with plastic bags, sealed and labelled with date of collection, location of collection [Global Positioning System (GPS) coordinate, postal code or address]. For domestic dog samples, the name and phone number of the dog owner was also noted. Two colon samples from carcasses of coyotes were submitted by local wildlife conservation officers who retrieved the colon from two unique animals. Samples were stored at $-80^{\circ} \mathrm{C}$ for at least 72 hours to inactivate E. multilocularis ova. The colonic samples were scraped on the mucosal surface to retrieve fecal matter.

\section{Laboratory testing}

Fecal material was stored and transported in sterile fecal containers, kept on ice, and sent with same day delivery to the reference laboratory (IDEXX Laboratories, Inc.) in Markham, Ontario. Fecal samples were processed immediately upon arrival and total nucleic acid extraction was performed using a KingFisher ${ }^{\mathrm{TM}}$ Flex Magnetic Particle Processor (Thermo Fisher Scientific, Vantaa, Finland) with proprietary lysis buffer and magnetic glass particles [Roche Diagnostics, Indianapolis, United States (US)]. Real-time polymerase chain reaction (PCR) assays were carried out using total nucleic acid purified from stool samples on a Roche LightCycler 480'M instrument using the manufacturer recommended cycling protocols and PCR reagents (23). Crossing points were calculated using the second derivative maximum-method analysis module with the high-sensitivity algorithm. Real-time PCR tests included PCR primers and a 6-FAM-TAMRA quenched conventional hydrolysis probe was adapted from Isaksson et al. (24).

The target gene for the real-time PCR tests was the mitochondrial gene $r r n L$. Six quality controls, including PCR positive and negative controls, negative extraction controls, environmental contamination control, spike-in internal positive control and pan-bacterial 16 ssrRNA internal sample control, were run to monitor for extraction efficiency, sample matrix inhibitors and cross contamination in the diagnostic runs. This PCR assay specific for Echinococcus species is in the process of being validated and peer reviewed. It was used in this study as it had successfully confirmed a clinical case of E. multilocularis of a dog in Ontario (personal communication January 11, 2019, Dr. Roxanne Chan, IDEXX Laboratories, Inc.).

\section{Analysis}

Data were stored in Microsoft Access and analyzed using Centers for Disease Control (Atlanta, Georgia, US) Epi-Info version 7.2.0.1 for descriptive statistics. Geocoding and mapping were done using Environmental Systems Research Institute ArcMap.

\section{Results}

In total, 169 samples were collected. This included 122 coyote scat samples, two coyote colon tissue samples, 44 domestic dog fecal samples, of which 34 samples were from unique animals collected directly from dog owners (the other 10 samples were collected from dog parks and may not necessarily be from unique animals), and one fox scat sample. 
Echinococcus multilocularis-positive samples were detected in nine $(10.6 \%)$ of 85 locations, with one positive sample in a suburban Winnipeg dog park and two positive samples in Birds Hill Provincial Park. The other samples appear to be more evenly dispersed across the more rural areas of the WMA. Figure 2 shows the sites where the samples were collected as well as the location of positive samples of E. multilocularis.

Figure 2: Map of the distribution of canid sample collection in the metropolitan area of Winnipeg, indicating Echinococcus multilocularis-positive samples

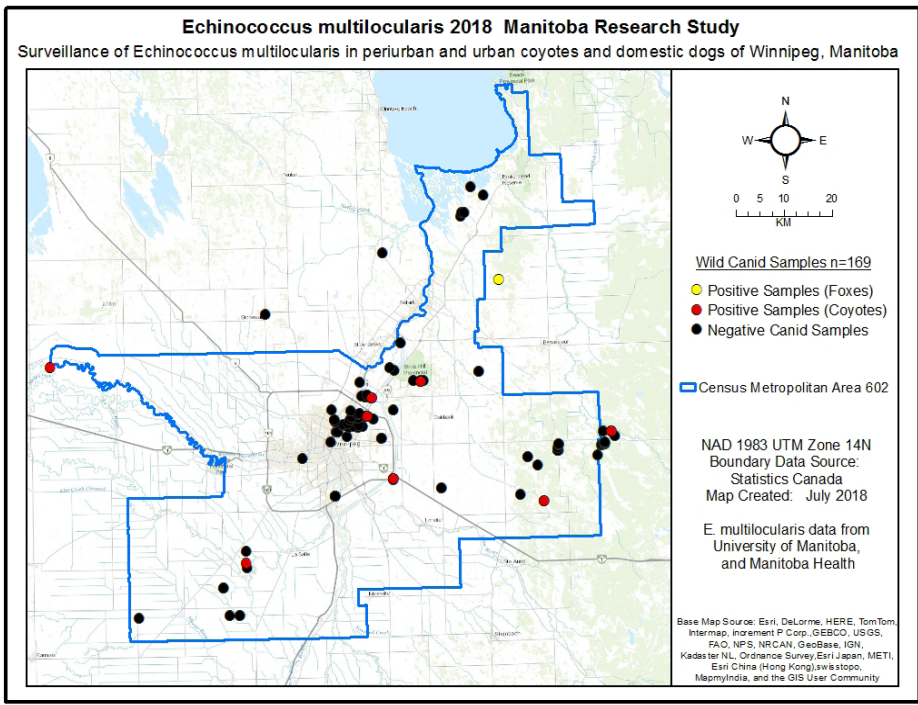

A red dot indicates an E. multilocularis-positive sample for coyote scat or tissue, a yellow dot indicates an E. multilocularis-positive sample for fox scat and a black dot indicates an E. multilocularis-negative canid sample. Blue outline depicts Census Metropolitan Area of Winnipeg; used with permission from Statistics Canada (22)

Of the samples sent for molecular processing, 10 (5.9\%) were positive for $E$. canadensis and 10 (5.9\%) were positive for E. multilocularis, with six (3.6\%) coinfections in five coyotes and one fox. E. multilocularis was found in $7.3 \%$ of all coyote samples, $0 \%$ of domestic canine samples and in the single fox sample. E. canadensis was found in $6.5 \%$ of all coyote samples, $2.3 \%$ of domestic dog samples and in the single fox sample (Table 1).

\section{Discussion}

This is the first confirmation of the presence of E. multilocularis in coyote feces in both urban and peri-urban areas of Winnipeg, Manitoba. The distribution of E. multilocularis in coyotes appears to be wide, with no hotspots. Although E. multilocularis was identified in a popular recreational provincial park and a dog park, no domestic dog samples were positive for this tapeworm. Only one case of the much more benign E. canadensis was found in a domestic dog sample.

Limitations of this study include the short duration of surveillance, possible repeated sampling from the same canids and the use of a molecular assay that is still undergoing peer review (23). No characteristics of the domestic dogs were collected, such as if they were outdoor dogs or consumed rodents. The wild canid scat sampling method was targeted and so does not reflect a uniform collection across the entire region. A much larger sample size and longer surveillance timeframe would be required to more fully map the extent of E. multilocularis canid infection in the Winnipeg metropolitan area.

\section{Implications and next steps}

Our findings indicate a risk of human and domestic dog exposure to coyote feces infected with E. multilocularis in the urban and peri-urban areas of Winnipeg.

It is not completely known how often human exposure to the parasite will result in infection leading to alveolar echinococcosis. In 2001, a five-year study in Switzerland was published that showed a high human seropositivity rate to $E$. multilocularis, with no increase in disease rate. One explanation was a potential increased immunity in this population (25). However, a 2007 review found a significant increase in the incidence of alveolar echinococcosis in Switzerland after 2000, and noted this had been preceded by an increase in this infection in the local fox population 10-15 years prior (26). It was thought this reflected the long clinical latency period of alveolar echinococcosis.

Table 1: Number and percent of canid samples infected with Echinococcus tapeworms in urban and peri-urban areas of Winnipeg, 2018

\begin{tabular}{|l|r|r|r|r|r|r|}
\hline \multirow{2}{*}{$\begin{array}{c}\text { Source of } \\
\text { stool sample }\end{array}$} & \multicolumn{4}{|c|}{ Echinococcus multilocularis } & \multicolumn{3}{c|}{ Echinococcus canadensis } \\
\cline { 2 - 8 } & \# of samples & \# positive stools & \% positive & \# of samples & \# positive stools & $\%$ positive \\
\hline Domestic dog & 44 & 0 & 0 & 44 & 124 & 1 \\
\hline Coyote & 124 & 9 & 7.3 & 100 & 1 & 1 \\
\hline Fox & 1 & 1 & 5.9 & 169 & 100 \\
\hline Total & 169 & 10 & 5.9 & 10 \\
\hline
\end{tabular}

Abbreviation: \#, number

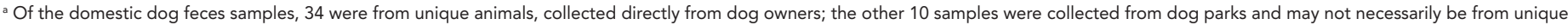
animals

${ }^{b}$ The 124 coyote scat samples are not necessarily from unique animals; hence, several samples could have originated from one coyote 
A longer study is now underway to determine if the high prevalence of E. multilocularis in the environment is associated with an increased incidence of alveolar echinococcosis in humans (25).

Based on the evidence of E. multilocularis in the environment in several provinces and the recent human cases in Alberta and Manitoba, further studies are indicated. To better characterize prevalence and geographic distribution, research with more locations and samples, spatial analysis and in-province molecular diagnostic capabilities are indicated. If warranted, a human seropositivity study of E. multilocularis in affected regions could be considered.

This emerging issue is particularly amenable to a One Health approach (27) involving physicians, veterinarians and wildlife experts. Ontario has made E. multilocularis infection in domestic dogs and humans reportable, in order to monitor the extent of the infection $(28,29)$. Although currently not notifiable elsewhere, it would be useful to report on the investigation of human alveolar echinococcosis cases, including any travel history (to confirm locally acquired disease versus imported from foreign countries) and information regarding the potential route of exposure to E. multilocularis ova. Public education concerning preventative measures to avert domestic dog and human infections may be indicated.

\section{Conclusion}

Echinococcus multilocularis has been documented in Canada for decades in the wild canid population, but human cases have been extremely rare. Over the last two decades, there has been a tendency for wild canids to migrate closer to urban populations, and there have been several recent human cases of alveolar echinococcosis caused by E. multilocularis. Although our study did not identify any cross contamination between coyotes and dogs in Winnipeg, the risk of exposure to E. multilocularis in urban and peri-urban settings suggests increased awareness and further study would help front line clinicians and public health officials be alert to and monitor this risk.

\section{Authors' statement}

CCKT - Project conception, literature searches, study design, field sampling, laboratory investigations, data analysis, writing (original draft, review, and editing)

JB - Project conception, study design, "in kind" laboratory investigations, software support, data analysis, writing (review and editing)

RR - Project conception, study design, software support, spatial data analysis, writing (review and editing)

DD - Project conception, study design, "in kind" laboratory support, data analysis, writing (review and editing) PJP — Project conception, literature searches, study design, field sampling support, data analysis, writing (review and editing)

\section{Conflict of interest}

None.

\section{Acknowledgements}

The authors wish to acknowledge Cadham Provincial Laboratory and the Parasitology Department for laboratory support, and D Berezanski and the Wildlife and Fisheries Branch (Manitoba Department of Sustainable Development) for assistance with sample collection and coyote education for volunteers. Many thanks to C Swiderek, Kilcona Dog Park Club and volunteers for help with sample collection. Our thanks to T Madill and $G$ Houghton for help with sample processing and sample collection and to IDEXX Laboratories Inc. for molecular analysis of the samples.

The results and conclusions are those of the authors and no official endorsement by Manitoba Health, Seniors and Active Living, or Manitoba Agriculture is intended or should be inferred.

\section{Funding}

This study received support for CCK Tse, who completed a Med 2 Summer Student Research Project from April to August 2018.

\section{References}

1. Catalano S, Lejeune M, Liccioli S, Verocai GG, Gesy KM, Jenkins EJ, Kutz SJ, Fuentealba C, Duignan PJ, Massolo A. Echinococcus multilocularis in urban coyotes, Alberta, Canada. Emerg Infect Dis 2012 Oct;18(10):1625-8. DOI PubMed

2. Eckert J, Deplazes P. Biological, epidemiological, and clinical aspects of echinococcosis, a zoonosis of increasing concern. Clin Microbiol Rev 2004 Jan;17(1):107-35. DOI PubMed

3. Moro P, Schantz PM. Echinococcosis: a review. Int J Infect Dis 2009 Mar;13(2):125-33. DOI PubMed

4. Food and Agriculture Organization of the United Nations and World Health Organization. Multicriteria-Based Ranking for Risk Management of Food-Borne Parasites. Geneva (Switzerland); WHO/FAO: 2014. www.fao.org/3/a-i3649e. pdf\%5Cnfiles/540/a-i3649e.pdf

5. Veit P, Bilger B, Schad V, Schäfer J, Frank W, Lucius R. Influence of environmental factors on the infectivity of Echinococcus multilocularis eggs. Parasitology 1995 Jan;110(Pt 1):79-86. DOI PubMed

6. Jenkins EJ, Castrodale LJ, de Rosemond SJ, Dixon BR, Elmore SA, Gesy KM, Hoberg EP, Polley L, Schurer JM, Simard M, Thompson RC. Tradition and transition: parasitic zoonoses of people and animals in Alaska, northern Canada, and Greenland. Adv Parasitol 2013;82:33-204. DOI PubMed 
7. Hildreth MB, Sriram S, Gottstein B, Wilson M, Schantz PM. Failure to identify alveolar echinococcosis in trappers from South Dakota in spite of high prevalence of Echinococcus multilocularis in wild canids. J Parasitol 2000 Feb;86(1):75-7. DOI PubMed

8. Brunetti E, Kern P, Vuitton DA; Writing Panel for the WHO-IWGE. Expert consensus for the diagnosis and treatment of cystic and alveolar echinococcosis in humans. Acta Trop 2010 Apr;114(1):116. DOI PubMed

9. Wilson JF, Rausch RL. Alveolar hydatid disease. A review of clinical features of 33 indigenous cases of Echinococcus multilocularis infection in Alaskan Eskimos. Am J Trop Med Hyg 1980 Nov;29(6):1340-55. DOI PubMed

10. Caire Nail L, Rodríguez Reimundes E, Weibel Galluzzo C, Lebowitz D, Ibrahim YL, Lobrinus JA, Chappuis F. Disseminated alveolar echinococcosis resembling metastatic malignancy: a case report. J Med Case Reports 2017 Apr;11(1):113. DOI PubMed

11. Ammann RW, Eckert J. Cestodes. Echinococcus. Gastroenterol Clin North Am 1996 Sep;25(3):655-89. DOI PubMed

12. Torgerson PR, Schweiger $A$, Deplazes $P$, Pohar M, Reichen J, Ammann RW, Tarr PE, Halkik N, Müllhaupt B. Alveolar echinococcosis: from a deadly disease to a well-controlled infection. Relative survival and economic analysis in Switzerland over the last 35 years. J Hepatol 2008 Jul;49(1):72-7. DOI PubMed

13. James E, Boyd W. Echinococcus Alveolaris: (With the Report of a Case). Can Med Assoc J 1937 Apr;36(4):354-6. PubMed

14. Samuel WM, Ramalingam S, Carbyn LN. Helminths in coyotes (Canis latrans Say), wolves (Canis lupus L.), and red foxes (Vulpes vulpes L.) of southwestern Manitoba. Can J Zool 1978 Dec;56(12):2614-7. DOI PubMed

15. Government of Manitoba. Living with Wildlife in Manitoba (Accessed 2018-08-03). www.gov.mb.ca/sd/wildlife/problem_ wildlife/coyote.html

16. Deplazes P, Hegglin D, Gloor S, Romig T. Wilderness in the city: the urbanization of Echinococcus multilocularis. Trends Parasitol 2004 Feb;20(2):77-84. DOl PubMed

17. Skelding A, Brooks A, Stalker M, Mercer N, deVilla E, Gottstein B, Peregrine AS. Hepatic alveolar hydatid disease (Echinococcus multilocularis) in a boxer dog from southern Ontario. Can Vet J 2014;55:551-553. PubMed

18. Trotz-Williams LA, Mercer NJ, Walters JM, Wallace D, Gottstein B, Osterman-Lind E, Boggild AK, Peregrine AS. Public health follow-up of suspected exposure to Echinococcus multilocularis in Southwestern Ontario. Zoonoses and Public Health 2017;64:460-467. DOI PubMed

19. Jenkins EJ, Peregrine AS, Hill JE, Somers C, Gesy K, Barnes B, Gottstein B, Polley L. Detection of European strain of Echinococcus multilocularis in North America. Emerg Infect Dis 2012 Jun;18(6):1010-2. DOI PubMed

20. Gesy K, Hill JE, Schwantje H, Liccioli S, Jenkins EJ. Establishment of a European-type strain of Echinococcus multilocularis in Canadian wildlife. Parasitology 2013 Aug;140(9):1133-7. DOI PubMed
21. Gesy KM, Jenkins EJ. Introduced and Native Haplotypes of Echinococcus multilocularis in Wildlife in Saskatchewan, Canada. J Wildl Dis 2015 Jul;51(3):743-8. DOl PubMed

22. Statistics Canada. 2017. Winnipeg [Census metropolitan area], Manitoba and Manitoba [Province]. Census Profile. 2016 Census. Statistics Canada Catalogue no. 98-316-X2016001. Ottawa (ON): Stats Can; November 29, 2017 (Accessed 2018-07-25). www12. statcan.gc.ca/census-recensement/2016/dp-pd/prof/index. $\mathrm{cfm}$ ? Lang $=\mathrm{E}$

23. Sindern N, Suchodolski JS, Leutenegger CM, Mehdizadeh Gohari I, Prescott JF, Proksch AL, Mueller RS, Busch K, Unterer S. Prevalence of Clostridium perfringens netE and netF toxin genes in the feces of dogs with acute hemorrhagic diarrhea syndrome. J Vet Intern Med 2019 Jan;33(1):100-5. DOI PubMed

24. Isaksson $M$, Hagström $\AA$, Armua-Fernandez MT, Wahlström $H$, Ågren EO, Miller A, Holmberg A, Lukacs M, Casulli A, Deplazes $P$, Juremalm M. A semi-automated magnetic capture probe based DNA extraction and real-time PCR method applied in the Swedish surveillance of Echinococcus multilocularis in red fox (Vulpes vulpes) faecal samples. Parasit Vectors 2014 Dec; 7:583. DOI PubMed

25. Gottstein B, Saucy F, Deplazes P, Reichen J, Demierre G, Busato A, Zuercher C, Pugin P. Is high prevalence of Echinococcus multilocularis in wild and domestic animals associated with disease incidence in humans? Emerg Infect Dis 2001 May-Jun;7(3):408-12. DOI PubMed

26. Schweiger A, Ammann RW, Candinas D, Clavien PA, Eckert J, Gottstein B, Halkic N, Muellhaupt B, Prinz BM, Reichen J, Tarr PE, Torgerson PR, Deplazes P. Human alveolar echinococcosis after fox population increase, Switzerland. Emerg Infect Dis 2007 Jun;13(6):878-82. DOI PubMed

27. One Health Initiative. About the One Health Initiative. (Accessed 2018-08-09). www.onehealthinitiative.com/about.php

28. Ontario Ministry of Health and Long-Term Care. Management of Echinococcus multilocularis Infections in Animals Guideline, 2018 (Accessed 2018-12-18). http://health.gov.on.ca/ en/pro/programs/publichealth/oph_standards/docs/ protocols_guidelines/Management_of_EM_Infections_in_ Animals_2018.pdf

29. Government of Ontario. R.R.O. 1990, Reg. 557: COMMUNICABLE DISEASES - GENERAL. Toronto (ON): Government of Ontario; 2017 (Accessed 2018-07-25). www. ontario.ca/laws/regulation/900557/v5 